\title{
ARTICLE
}

Received 28 Nov 2012 | Accepted 24 May 2013 | Published 19 Jun $2013 \quad$ DOl: 10.1038/ncomms3054

\section{Dipolar-energy-activated magnetic domain pattern transformation driven by thermal fluctuations}

\author{
M. Kronseder ${ }^{1}$, M. Buchner ${ }^{1}$, H.G. Bauer ${ }^{1} \&$ C.H. Back ${ }^{1}$
}

Two-dimensional ferromagnetic layers can serve as a playground for the study of basic physical properties of various pattern forming systems by virtue of their tuneable magnetic properties. Here we use threshold photoemission magnetic circular dichroism in combination with photoemission electron microscopy to investigate ultra-thin ferromagnetic $\mathrm{Fe} / \mathrm{Ni} / \mathrm{Cu}(001)$ films in the stripe domain phase near the spin reorientation transition as a function of film thickness, temperature and effective anisotropy. Here we report a metastable domain state with domain width larger than the thermodynamically stable one as a result of a rapid reduction of the anisotropy. The transformation into the equilibrium state takes place via the propagation of a transition front, which originates from defined steps in the film thickness.

\footnotetext{
${ }^{1}$ Physics Department, Universität Regensburg, Universitätsstr, 31, 93040 Regensburg, Germany. Correspondence and requests for materials should be addressed to M.K. (email: matthias.kronseder@ur.de).
} 
T he formation of intricate domain patterns on mesoscopic as well as macroscopic length scales is connected with the competition between short-range attractive and long-range repulsive interactions. In the particular case studied heremagnetic domain patterns in ultra thin ferromagnetic films-the basis for pattern formation is the interplay between various magnetic energies where the ratio between uniaxial anisotropy, dipolar and exchange interaction plays the decisive role. Magnetic domain patterns in perpendicularly magnetized ultra thin films are typically characterized by the geometric shape of the domains, such as labyrinth, stripe, bubble or simply disordered patterns ${ }^{1-5}$. According to theory and experiments, these patterns appear in distinct sequences as a function of temperature or film thickness. What is more, these twodimensional magnetic systems may exhibit a spin reorientation transition (SRT) where the magnetization vector $\mathbf{M}$ rotates from out-of-plane into the plane of the film. When approaching the SRT from the perpendicularly magnetized region of the magnetic film, the domain width becomes narrower and finally assumes a minimum value-independent of the geometrical shape of the domain pattern-before $\mathbf{M}$ finally rotates into the plane ${ }^{6}$. In essence, in thermal equilibrium, the domain width in the phase with perpendicular magnetization adapts to minimize the free energy, which is dominated by exchange, anisotropy and dipolar energy, which are, in turn, functions of temperature and film thickness. As a consequence of the change of the equilibrium domain width when approaching the SRT, additional domains have to be introduced into the domain pattern, which involves domain wall displacement driven by thermal energy.

In any real system, the energy landscape shows local corrugation caused by imperfections of the substrate or the ferromagnetic film or arising from self-generated defects in the domain pattern. It is due to these ripples in the energy landscape that pattern transformations, initiated by the change of, for example, the equilibrium domain width, are in general stepwise reactions driven by thermal fluctuations. Transitions can also be driven by changing external parameters. In this case, ripples in the energy landscape remain observable as long as the driving external parameter is of the same order of magnitude as the thermal fluctuations. In the case of tuning the externally applied magnetic field the corrugated energy landscape is evidenced by the well-known Barkhausen jumps.

As for any statistical process, thermally driven transitions have a certain finite rate of change determined by the thermal energy and the local corrugation of the energy landscape. As a consequence, after a parameter changes faster than the systems inherent relaxation time, which is determined by thermal fluctuations, a metastable state in which the system may remain for an unpredictably long time emerges. For the transition back into the equilibrium state, a local activation across a certain energy barrier may be sufficient, which eventually initiates a global transition. A famous manifestation of such a transition is the formation of a condensation front in supercooled liquids ${ }^{7,8}$.

In this article, we investigate the emergence of a metastable magnetic state as well as its stepwise transformation into equilibrium for the out-of-plane magnetized two-dimensional system $\mathrm{Fe} / \mathrm{Ni} / \mathrm{Cu}(001)$. Here, we induce a magnetic metastable state at low temperatures-connected to reduced thermal fluctuations-by rapidly changing the ratio of the magnetic energy contributions, which are responsible for the equilibrium domain width. A global transition driven by a magnetic condensation front is imaged using a unique non-stroboscopic imaging technique, after the transition has been initialized by a rare event.

\section{Results}

Experimental and system properties. The system $\mathrm{Fe} / \mathrm{Ni} / \mathrm{Cu}(001)$ exhibits a stable out-of-plane phase and shows a wealth of domain patterns ${ }^{9}$. The equilibrium domain width of the system is determined by the temperature and thickness-dependent ratio of the anisotropy, dipolar and exchange coupling constants. Consequently, the domain width depends on the two parameters temperature $T$ and film thickness $d$. These parameters can be condensed to an effective temperature $T^{*}(d, T)$ of the magnetic system ${ }^{9}$, which is a characteristic feature for the classification of the magnetic domain state ${ }^{4}$. A full phase diagram of the magnetic domain configurations for this system is given in ${ }^{10,11}$. In the vicinity of the SRT, the effective temperature of the system increases, which leads to an evolution of the domain pattern with an exponentially decreasing domain width. The domain width finally reaches a finite value at the SRT. Consequently, when changing the effective temperature, the domain pattern has to adapt by inserting or eliminating domains, where each single domain wall displacement must be thermally driven. Thus, when rapidly changing the ratio of the coupling constants while simultaneously suppressing thermal fluctuations by cooling the system to low temperature, the system is unable to adapt to the new equilibrium domain configuration. In other words, in the absence of large thermal fluctuations the system cannot leave the initial state and cannot gradually condensate into its new equilibrium state. However, a local transition may take place in regions where the final state is still energetically reachable even with reduced thermal fluctuations or by the occurrence of rare events. This initialization, in turn, leads to the emergence of a transition front gradually moving across the system. For supercooled water such a transition front is known as the condensation front ${ }^{7}$. In our case, the system forms well-separated regions with large and small domain width. As in the case of supercooled liquids, where the crystallization front affects the surrounding liquid molecules by absorbing their kinetic energy, the magnetic transition front lowers the local energy in front of the transition region by reducing the long-range dipolar energy due to a higher domain wall density behind the front.

Thickness-dependent domain width evolution. Threshold photoemission magnetic circular dichroism (TP-MCD) ${ }^{12,13}$ in combination with photoemission electron microscopy (PEEM) has been used to investigate the magnetic structure. As the work function of $\mathrm{Fe}$ and $\mathrm{Ni}$ is around $\sim 5 \mathrm{eV}$ and the used photon energy is $3.06 \mathrm{eV}$, Cs adatoms were deposited before PEEM operation in order to lower the work function and, hence, to increase the photocurrent. Cs adatom deposition affects the magnetic surface anisotropy such that the SRT is shifted to lower Fe thicknesses. The reason for the shift of the SRT is the modification of the magnetic surface anisotropy, which depends on the chemical composition of the surface layer. Cs deposition changes the occupation of the atomic orbitals of the surface layer owing to hybridization ${ }^{14,15}$. However, the domain patterns and the evolution of the patterns relative to the SRT remain unaffected motivating the introduction of an effective temperature, where the real temperature as well as the thickness are incorporated on an equal footing.

Typical images acquired using TP-MCD PEEM are shown in Fig. 1a,b. Images are obtained on wedge-type samples using an exposure time of $12 \mathrm{~ms}$. Between the acquisition of images recorded with left and right circularly polarized light about $\sim(10-30) s$ are required to read out the camera and to change the circularity of the light. We show difference images (left-right) normalized to the sum and identify the SRT in the centre of the images. Figure 1c shows the domain width evolution versus the 
a

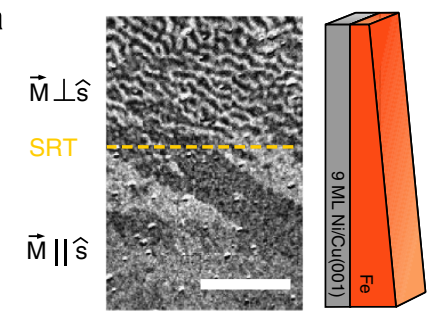

C

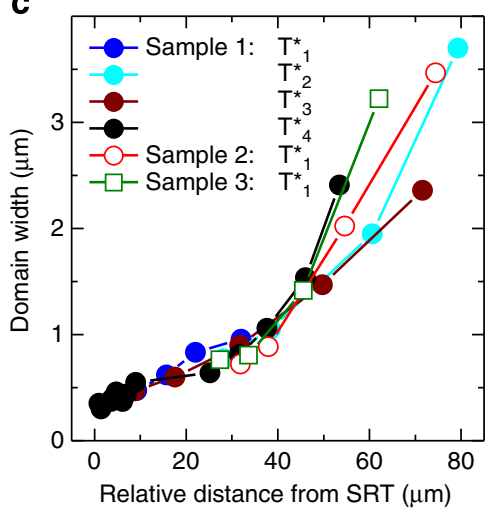

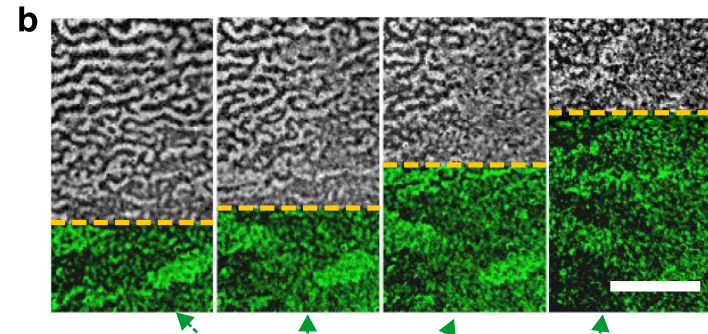

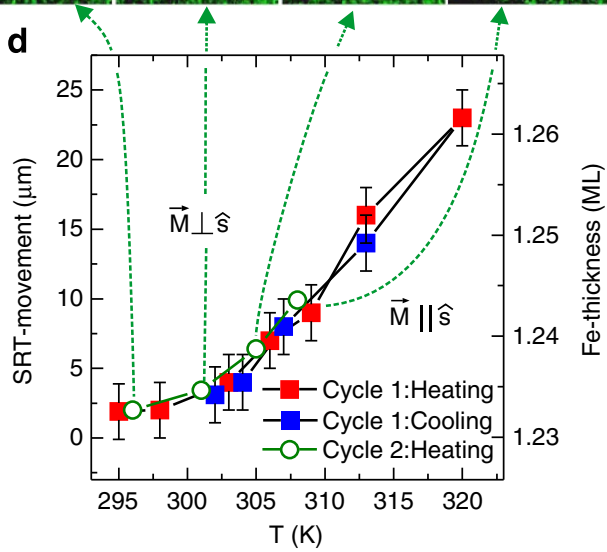

Figure 1 | Film thickness and temperature dependence of the domain width evolution. (a) TP-MCD PEEM image of the domain evolution at a wedge as depicted in the schematic drawing, where in the upper part of this image the magnetization is perpendicular to the surface ( $\hat{s}$ is a vector parallel to the surface) and in the lower part the sample is in-plane magnetized. The dashed line indicates the SRT. (b) Images taken during a heating cycle at 296, 301, 305 and 308 K. (c) Domain width evolution with decreasing distance from the SRT for one sample with different effective anisotropies (sample 1) as well as for individual samples with different thickness slopes (samples 2, 3). The distances to the SRT are re-scaled to the slope of the wedge of sample 1. (d) Motion of the SRT towards the out-of-plane phase while increasing the temperature. Scale bar, $10 \mu \mathrm{m}$.

distance to the SRT, where a positive distance means a thinner film thickness. The graph summarizes the dependence for different samples as well as for a single sample, but at different effective temperatures due to a change of the effective anisotropy by Cs adatoms. The main result that can be extracted from this figure is that the overall dependence of the domain width with respect to the distance to the SRT remains similar, which is in agreement with Won et al. ${ }^{9}$ and Abe et al. ${ }^{11}$ The film thickness dependence of the domain width is mainly caused by the variation of the effective anisotropy with film thickness, which can be written as $K_{\mathrm{e}}=\left(K_{\mathrm{B}}-\frac{1}{2} \mu_{0} M_{\mathrm{S}}^{2}\right)+\left(K_{\mathrm{S}}+K_{\mathrm{I}}\right) / d(\operatorname{refs} 10,16)$. Here $K_{\mathrm{B}}$ is the bulk anisotropy, $M_{\mathrm{S}}$ is the magnetization at saturation, $K_{\mathrm{S}}$ and $K_{\mathrm{I}}$ are the surface and interface anisotropies, respectively, and $d$ is the film thickness. For $K_{\mathrm{e}}>0$, the system favors out-of-plane magnetization and at $K_{\mathrm{e}}=0$ the SRT occurs. As has been shown by Won et al. ${ }^{9}$, the equilibrium stripe domain width is an exponential function of the effective magnetic anisotropy $K_{\mathrm{e}}$, the exchange interaction $J$ and the dipolar energy $\Omega$. All energy terms are assumed to be average quantities with respect to the whole magnetic stack. In addition, the exchange interaction is assumed to depend on film thickness, $J=J(d)$. Based on the dependence of $K_{\mathrm{e}}$, it is clear that any change of the chemical composition of the sample surface, for example, due to deposition of Cs adatoms, leads to a constant shift of the whole SRT, but leaves the overall domain width dependence unchanged.

Temperature-dependent domain width evolution. In addition to being thickness dependent, all microscopic coupling constants in general also depend on temperature ${ }^{17}$. Therefore, we also measured the temperature dependence of the SRT position, which is shown in Fig. 1b,d. The SRT was extracted from images with out-of-plane as well as in-plane contrast, Fig. 1b. In these images, the Fe thickness increases from top to bottom. In addition to the shift of the SRT, we can observe the formation of in-plane domains (coloured in green). In-plane contrast can easily be determined from the images due to the formation of larger irregular domains, as shown in Fig. 1a. This is in agreement with the theoretically predicted phase diagram by Cannas et al. ${ }^{18}$ These authors showed that only in a narrow temperature and effective anisotropy interval, a paramagnetic phase exists between the ferromagnetic out-of-plane and in-plane phase. The existence of some fast moving domains near the SRT (image exposure time $12 \mathrm{~ms}$ ), as already observed in $^{9}$, is confirmed in these images as we observe zero contrast locally but within the perpendicular stripe domain phase. Surprisingly, Fig. 1d shows almost the same evolution as the thickness dependence in Fig. 1c signifying that the interplay of $K_{\mathrm{e}}(T), J(T)$ and $\Omega(T)$ leads to a temperature dependence comparable to an effective thickness dependence in the vicinity of the SRT. This is in agreement with the evolution of the domain width as a function of the real temperature measured in ref. 19. Summarizing, these facts (Fig. 1c,d) show that temperature and thickness affect the domain width and therefore the domain pattern in the same manner. Hence, the introduction of an effective temperature $T^{*}(d, T)$, implicitly depending on film thickness $d$ and real temperature $T$ is reasonable. The equilibrium domain width then depends on $T^{*}(d, T)$.

In addition, a change of the effective anisotropy due to Cs deposition can be taken into account by introducing the effective temperature $T_{\mathrm{SRT}}^{*}$ at which the SRT occurs. A variation of the effective anisotropy, which is neither caused by a change of the thickness $d$ nor caused by a change of the temperature $T$ can therefore be expressed by a variation of this effective SRTtemperature shifting also the whole domain width evolution. Based on these parameters $\left(T^{*}(d, T), T_{\mathrm{SRT}}^{*}\right)$ a comprehensive 
model for the equilibrium and non-equilibrium evolution of the domain width near the SRT can be derived leading to a description of the experimentally measured transition front, which is the main purpose of this article.

As the domain width depends on $K_{\mathrm{e}}(T), d$ and $T$ one may ask the questions: how might the domain width evolve upon a sudden change of $K_{\mathrm{e}}(T)$ at reduced temperatures? To what extent do thermal fluctuations determine the ability of a system to undergo a transition into a different state? In other words, what happens if a system is abruptly driven into an energetically unfavourable state while simultaneously suppressing thermal fluctuations? The result is a metastable state, in our case a state with wide domains.

Transition front velocity. The investigated stepped wedge $\mathrm{Fe} / \mathrm{Ni} /$ $\mathrm{Cu}(001)$ film exhibits large modifications of the domain width at the individual thickness steps $(0.2$ or $0.4 \mathrm{ML})$ of the Fe layer, which are equally spaced by $\sim(0.9-1.1) \mathrm{mm}$. Before cooling the sample to about $180 \mathrm{~K}$, a small amount of Cs (surface coverage $<\sim 0.1$, see methods section) has been deposited, to be able to image the domain evolution at the individual steps of the sample. The cooling process shifts the SRT point towards the in-plane region, that is, stabilizes the out-of-plane phase which is equivalent to reducing the effective temperature $T^{*}(d, T)$ in our model. To drastically reduce the effective uniaxial anisotropy we deposit additional Cs for $\sim 5 \mathrm{~min}$ (corresponding to $<0.02$ change of Cs coverage), which reduces the surface anisotropy term $K_{\mathrm{S}}$. At room temperature, reducing $K_{\mathrm{S}}$ leads to a shift of the critical film thickness at which the SRT occurs of more than $0.2 \mathrm{ML}$. However, at low temperatures this is not observed. The system remains in the initial state at all terraces of the stepped wedge sample for a random period of time (15-40 min), until, starting at a step of the film thickness, smaller domains spread out of larger domains, see the insets of Fig. 2. Three features can be extracted from this sequence of images. First, there is a clear front with finite width of smaller domains slowly moving across the sample. Second, all spreading domains have almost equal domain width. And third, only very few domains with the 'final' domain width emerge at larger distances from the transition front (some hundreds of micrometres) but still in its vicinity. When the position of the transition front is plotted as a function of time, a straight line results, leading to a velocity of $\sim 3.0 \mu \mathrm{m} \mathrm{min}{ }^{-1}$.

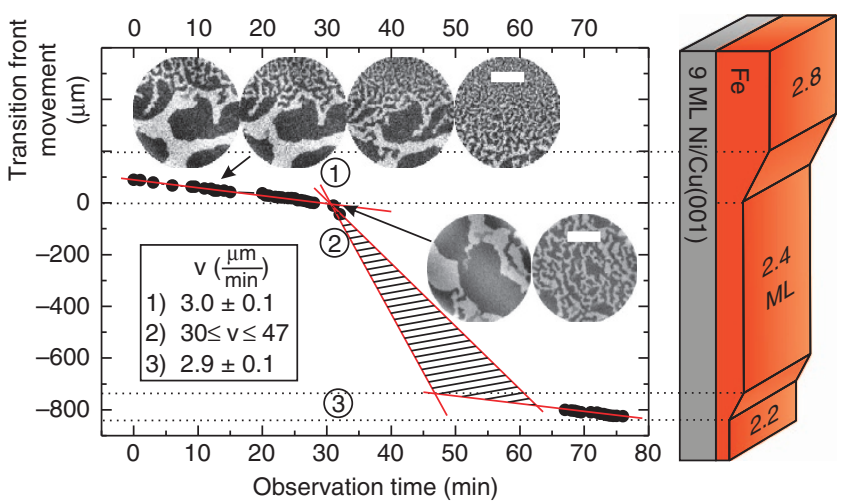

Figure 2 | Observation of the movement of the transition front. The movement of the main transition front as a function of observation time is shown. The error bars are much smaller than the symbols. Three regions with individual transition front velocities can be recognized, which correspond to a region with constant Fe thickness (region 2 ) and regions with varying $\mathrm{Fe}$ thickness (regions 1 and 3 ). The images show the domain patterns used to determine the transition front position. In region 2 only two images could be obtained, which resulted in a transition front velocity faster than the field of view divided by the acquisition time for both images. Scale bar, $10 \mu \mathrm{m}$.
Surprisingly, this velocity is about one order of magnitude larger in regions with constant film thickness. At each step in film thickness, the velocity returns to the lower value. Owing to the small field of view of the PEEM we acquired only two images (from the same position on the sample) in the region of a terrace leading to a large unknown region, represented by the hatched area in Fig. 2. Consequently, a large error in the determination of the velocity of the transition front arises. However, as we are able to map the position of the transition front onto the layer thickness by analysis of the change of the domain width induced by thickness variations, the transition front evolution can be restricted to the hatched area in Fig. 2. Similar transition front velocities have been observed in five different samples.

\section{Discussion}

The experimentally measured and theoretically predicted ${ }^{2,9}$ domain width dependence on the effective temperature $T^{*}(d, T)$ results in an exponential evolution. This is shown in Fig. 3a,b. Hence, for a given effective temperature $T^{*}(d, T)$ a certain domain width $w_{\mathrm{D}}$ results. Furthermore, a change of the effective anisotropy can be expressed by a shift of the effective SRT temperature $T_{\mathrm{SRT}}^{*}$. Hence, a reduction of the effective anisotropy by Cs deposition reduces $T_{\mathrm{SRT}}^{*}$ and consequently accompanies a reduction of the equilibrium domain width for a given effective temperature $T^{*}(d, T)$. Both results are in agreement with our experiments. In case of an abrupt, large change of the effective anisotropy the system is unable to adapt to the new equilibrium domain width $w_{\mathrm{D}}$ and leads to the metastability of the initial state at $w_{\mathrm{D}}^{\text {old }}$. In Fig. $3 \mathrm{c}$, both the equilibrium (black) as well as the non-equilibrium (grey) domain width evolution is plotted. A gradual transition towards the equilibrium domain width is suppressed due to a large gap between these states separated by an energy barrier when the system is kept at $T^{*}(d, T)$.

Corrugation of the energy landscape, caused by either structural imperfections or self-generated glassiness ${ }^{8,20}$, renders any domain pattern transformation into a gradual step-by-step process. At low temperatures, the thermal energy in the system may not suffice to overcome the individual energy barriers of the corrugated energy landscape. Hence, the corrugated energy landscape can be regarded as an effective global energy barrier retaining the system in the metastable state. It is astonishing that this local corrugation, which constitutes individual energy barriers for each single-domain wall motion (which is typically in the $\mu \mathrm{m}$-range), is able to keep a magnetic domain pattern with a domain width of some tens to hundreds of microns in a metastable state on macroscopic length scales (the sample width is in the mm-range). From this observation, one can conclude that the overall corrugated energy landscape has to be rather flat on average. At larger effective temperatures $T^{*}(d, T)$, the gap between the initial and final state becomes smaller and the transition becomes possible, as can be seen in Fig. 3d indicated by the red line.

An explanation for the observed transition front can be given in terms of our model by dividing the process into two parts. The first is the activation process, which is the initialization of the transition front by random nucleation of small domains somewhere in the sample. Once nucleation has taken place, the second process is the transport of the activation energy, eventually leading to the movement of the transition front. The activation process can be induced either by a rare event in the sense of an extremely large thermal fluctuation changing the domain state in a sufficiently large area or simply by a large enough film thickness variation. In the latter case, the domain width decreases with increasing thickness and the transition from the initial to the final state occurs, as shown in Fig. 3d. The actual domain width 

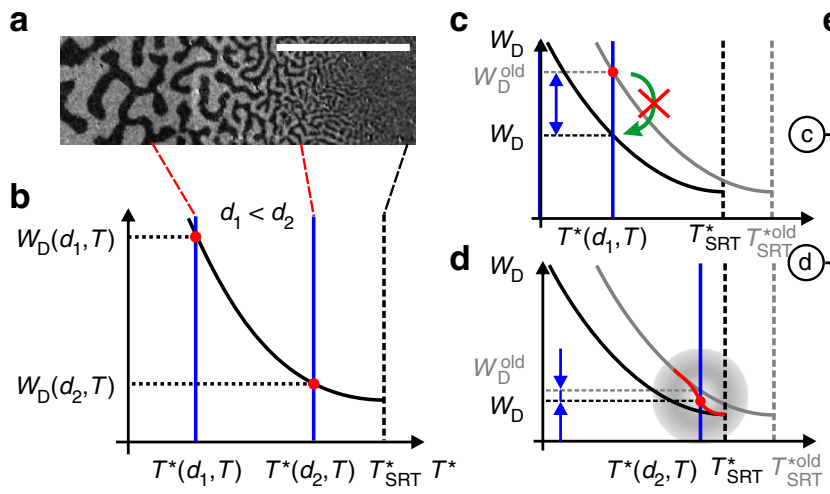

e

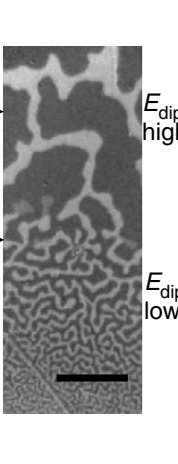

$\mathbf{f}$

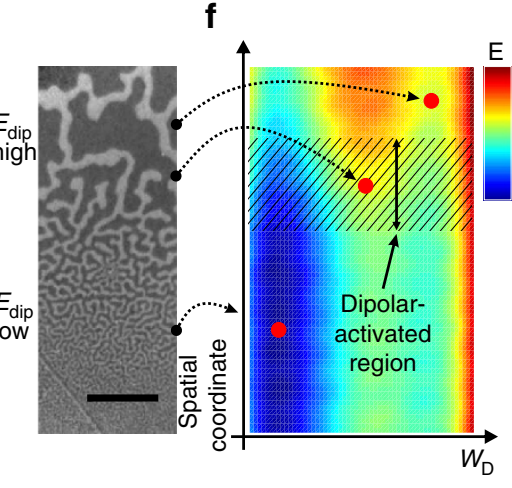

Figure 3 | Model for the equilibrium and non-equilibrium domain width evolution and application to the transition front measurement. (a) Shows a typical domain pattern in the vicinity of the SRT where the Fe thickness increases from left to right by about 0.14 ML. As discussed in Fig. 1, the domain width shows an exponential dependence on the effective temperature $T^{*}(d, T)$, depending on the thickness $d$, temperature $T$ and effective anisotropy, which can comprehensively be plotted as shown in (b). Cs adatoms, for instance, decrease the effective SRT temperature $T_{\text {SRT }}^{* \text { old }} \rightarrow T_{\text {SRT }}^{*}$ thereby shifting the whole domain width evolution as shown in (c,d). At low effective temperature, a large gap between the thermally reachable initial and final states opens up. Owing to the exponential evolution of the domain width the gap becomes narrower at larger effective temperatures as shown in (d) and makes the transition from the former (grey line) to the new (black line) domain width possible, which represents the activation process. The curve connecting both lines (red curve) represents the actual evolution. Images in (e) show such a transition front, where the time duration between these images was $\sim 16$ min. These images also show the continuous decrease of the domain width behind the transition front, as predicted by the model. In (f), a schematic dependence of the energy landscape on the domain width and a spatial coordinate map is shown. During the transition to the smaller domain width, the dipolar energy $E_{\text {dip }}$ decreases, which further decreases the energy of the domain configuration in particular in the vicinity of the transition. The red dots in (b-d,f) represent the actual state of the system. Scale bar, $25 \mu \mathrm{m}$.

evolution is represented by the red curve connecting the non-equilibrium and equilibrium domain width evolution. The transport of the activation energy, that is, the translation of the transition front, is based on the reduction of the long-range dipolar energy after the first step of the transition has occurred.

In Fig. 3f, a schematic dependence of the energy landscape in the vicinity of the transition front is shown. A spatial coordinate parallel to the propagation direction of the transition front is plotted on the left axis and the other axis represents the domain width. The energy level is given by the colour scale. The hatched area represents the region of the transition front. Above the hatched area, the system (red dots) is in the metastable state (large domain width), whereas below the hatched area the system is already in the equilibrium state, just as in the real images Fig. 3e. The dipolar energy of the system is given by the actual state of the system and is reduced when the system transforms into a state with smaller domains, hence, further reducing the global energy minimum, left part of Fig. 3f. A micromagnetic simulation (The code is available at http://math.nist.gov/oommf) supports this argument, as the dipolar energy density, extracted from simulations of realistic domain configurations, is about thirteen times smaller for the smaller domain width configuration than for the large domain state. The initial configurations for the two simulations were extracted from measured images, that is, one before the transition front, with a domain width $w_{\mathrm{D}}=6.8 \mu \mathrm{m}$, and the other after the transition front $w_{\mathrm{D}}=2.1 \mu \mathrm{m}$. Hence, the domain state in the upper region of Fig. 3e has a characteristically higher dipolar energy $E_{\text {dip }}$ than the lower part exhibiting small domains. Owing to the long but finite interaction range of the dipole interaction, this additional reduction affects the domain states only in the vicinity of the transition front, as shown by the hatched area in Fig. 3f. Hence, this could be an explanation for the reduction of the energy barrier between the initial and final domain state, which eventually makes the transition possible. In addition, the transition front velocity is mainly determined by the strength of thermal fluctuations as well as the corrugation of the energy landscape. Each single domain wall displacement occurs from pinning site to pinning site resulting in a distinct transition front velocity. Note that it is only due to the corrugation of the energy landscape that this transition is a continuous one. However, the nature of the different but reproducible velocities for regions with varying (regions 1 and 3 in Fig. 2) and with constant thickness (region 2 in Fig. 2) is rather unknown. Applying our model in regions with decreasing effective temperature (in this case decreasing thickness), the gap between the initial and final state opens up while the transition front moves and thereby leads to an increasing relaxation time into the equilibrium state. In regions with constant film thickness, the energy gap stays constant. Hence, the transition front velocities must be different for these regions.

In summary, we have employed a novel laboratory-based microscopy technique capable of high speed non-stroboscopic imaging of magnetic domains in ultra thin ferromagnetic films with large magnetic contrast. The method opens up new opportunities for the investigation of non-repetitive phenomena, for example, in the vicinity of magnetic instabilities. Here, a transition from a metastable magnetic domain state in the domain pattern of ultra thin $\mathrm{Fe} / \mathrm{Ni} / \mathrm{Cu}(001)$ films has been observed, where the metastability is induced by both corrugation of the energy landscape and suppressed thermal fluctuations. The emergence of a transition front slowly moving across the sample can be attributed to thermal fluctuations of the domain pattern and the long range but finite influence of the reduced dipolar energy at the leading edge of the transition front. A model based on the experimentally measured domain width evolution is derived and successfully applied to the intricate situation of this transition front. However, to fully predict the temporal evolution of the domain patterns in this complex magnetic model system detailed theoretical models and further experiments on even shorter time scales are required. In principle, the method presented in this paper can be extended to sub-microsecond time scales and might become interesting in other fields such as 
spintronics where non-stroboscopic imaging techniques are lacking, but necessary. A prominent example is the determination of domain wall velocities in current-induced domain wall motion experiments ${ }^{21}$.

\section{Methods}

Sample preparation. The experiments are conducted on a $\mathrm{Cu}(001)$ single-crystal disk cleaned by cycles of Ar-ion sputtering at $\sim(0.8-1) \mathrm{keV}$ and subsequent annealing at $\sim 800 \mathrm{~K}$. Fe and $\mathrm{Ni}$ thin films are deposited onto the $\mathrm{Cu}(001)$-crystal by means of electron-beam evaporation. The deposition rate for $\mathrm{Fe}$ is

$\sim 0.43 \mathrm{ML} \mathrm{min}^{-1}$ and for $\mathrm{Ni} \sim 0.34 \mathrm{ML} \mathrm{min}^{-1}$, both are controlled by measuring the molecular flux as well as by reflection high energy electron diffraction oscillations. The Ni thickness was in all cases kept constant at $9 \mathrm{ML}$, whereas the $\mathrm{Fe}$ film was grown either as a continuous or as a stepped wedge, with a thickness ranging from 0.8 to $2.4 \mathrm{ML}$.

Experimental method. The illumination unit of the TP-MCD PEEM setup comprises a $405 \mathrm{~nm}(3.06 \mathrm{eV})$ laser diode with an optical output power of $\sim(100-200) \mathrm{mW}$, a linear polarizer and a rotatable quarter-wave plate. The work function of the investigated samples is lowered by depositing Cs adatoms onto the sample surface during PEEM operation. According to Papageorgopoulos and $\mathrm{Chen}^{22,23}$, the maximum coverage of the surface by $\mathrm{Cs}$ adatoms is $\sim 0.3$, as above this coverage the sticking coefficient becomes zero. For the photon energy used a Cs coverage of $\sim 0.06$ is sufficient to extract photoelectrons. A larger surface coverage leads to larger photocurrents.

Simulation parameters. Simulations have been performed using the OOMMF code with the following parameters: exchange constant $A=2 \times 10^{-11} \mathrm{~J} \mathrm{~m}^{-1}$, saturation magnetization $M_{\mathrm{S}}=7.7 \times 10^{5} \mathrm{~A} \mathrm{~m}^{-1}$, uniaxial anisotropy $K_{\mathrm{e}}=3.848$ $\times 10^{5} \mathrm{~J} \mathrm{~m}^{-3}$, and cell size $c=10 \times 10 \times 1.79 \mathrm{~nm}^{3}$.

\section{References}

1. Abanov, Ar., Kalatsky, V., Pokrovsky, V. L. \& Saslow, W. M. Phase diagram of ultrathin ferromagnetic films with perpendicular anisotropy. Phys. Rev. B 51, 1023-1038 (1995).

2. Saratz, N. et al. Experimental phase diagram of perpendicularly magnetized ultrathin ferromagnetic films. Phys. Rev. Lett. 104, 077203 (2010).

3. Vaterlaus, A. et al. Two-step disordering of perpendicularly magnetized ultrathin films. Phys. Rev. Lett. 84, 2247-2250 (2000).

4. Portmann, O., Vaterlaus, A. \& Pescia., D. An inverse transition of magnetic domain patterns in ultrathin films. Nature 422, 701-704 (2003).

5. Kashuba, A. \& Pokrovsky., V. Stripe domain structures in a thin ferromagnetic film. Phys. Rev. B 48, 10335 (1993).

6. Kwon, H. Y. et al. A study of the stripe domain phase at the spin reorientation transition of two-dimensional magnetic system. J. Magn. Magn. Mater. 322, 2742-2748 (2010).

7. Mishima, O. \& Eugene Stanley, H. The relationship between liquid, supercooled and glassy water. Nature 396, 329-335 (1998).

8. Debenedetti, P. G. \& Stillinger, F. H. Supercooled liquids and the glass transition. Nature 410, 259-267 (2001).

9. Won, C. et al. Magnetic stripe melting at the spin reorientation transition in $\mathrm{Fe} /$ Ni/Cu(001). Phys. Rev. B 71, 224429 (2005).
10. Abe, H. et al. Spin reorientation transitions of $\mathrm{Fe} / \mathrm{Ni} / \mathrm{Cu}(001)$ studied by using the depth-resolved X-ray magnetic circular dichroism technique. J. Magn. Magn. Mater. 302, 86-95 (2006).

11. Choi, J. et al. Magnetic bubble domain phase at the spin reorientation transition of ultrathin $\mathrm{Fe} / \mathrm{Ni} / \mathrm{Cu}(001)$ film. Phys. Rev. Lett. 98, 207205 (2007).

12. Kronseder, M. et al. Threshold photoemission magnetic circular dichroism of perpendicularly magnetized $\mathrm{Ni}$ films on $\mathrm{Cu}(001)$ : theory and experiment. Phys. Rev. B 83, 132404 (2011).

13. Nakagawa, T. \& Yokoyama, T. Magnetic circular dichroism near the Fermi level. Phys. Rev. Lett. 96, 237402 (2006).

14. Callcott, T. A. \& Mac Rae, A. U. Photoemission from clean an cesium-covered nickel surfaces. Phys. Rev 178, 966-978 (1969).

15. Lindgren, S. Å. \& Walldén, L. Cu surface state and Cs valence electrons in photoelectron spectra from the $\mathrm{Cu}(111) / \mathrm{Cs}$ adsoprtion system. Solid State Commun. 28, 283-286 (1978).

16. Millev, Y. \& Kirschner, J. Reorientation transitions in ultrathin ferromagnetic films by thickness- and temperature-driven anisotropy flows. Phys. Rev. B 54, 4137-4145 (1996).

17. Kuch, W. et al. Thermal melting of magnetic stripe domains. Phys. Rev. B 83, 172406 (2011)

18. Cannas, S. A., Michelon, M. F., Stariolo, D. A. \& Tamarit, F. A. Interplay between coarsening and nucleation in an Ising model with dipolar interactions. Phys. Rev. B 77, 134417 (2008).

19. Portmann, O., Vaterlaus, A. \& Pescia, D. Observation of stripe mobility in a dipolar frustrated ferromagnet. Phys. Rev. Lett. 96, 047212 (2006).

20. Dzero, M., Schmalian, J. \& Wolynes, P. G. Glassiness in uniformly frustrated systems. Preprint at http://arxiv.org/abs/1011.2261 (2011).

21. Thomas, L. et al. Oscillatory dependence of current-driven magnetic domain wall motion on current pulse length. Nature 443, 197-200 (2006).

22. Papageorgopoulos, C. A. \& Chen, J. M. Coadsorption of cesium and oxygen on $\mathrm{Ni}(100)$ : I. Cesium probing of $\mathrm{NiO}$ bonding. Surf. Sci. 52, 40-52 (1975).

23. Papageorgopoulos, C. A. \& Chen, J. M. Cesium enhanced oxidation of $\mathrm{Ni}(100)$. Solid State Commun. 13, 1455-1457 (1973).

\section{Acknowledgements}

We acknowledge Alessandro Vindigni, Danilo Pescia, Christoph Strunk and Eric R. J. Edwards for fruitful discussions.

\section{Author contributions}

C.H.B. and M.K. designed the research; M.K. and M.B. prepared the samples, performed experiments; M.K., M.B. and C.H.B. analysed and interpreted the results; H.G.B. performed the simulations; M.K. developed the model; and M.K. and C.H.B. wrote the manuscript.

\section{Additional information}

Competing financial interests: The authors declare no competing financial interests.

Reprints and permission information is available online at http://npg.nature.com/ reprintsandpermissions/

How to cite this article: Kronseder, M. et al. Dipolar-energy-activated magnetic domain pattern transformation driven by thermal fluctuations. Nat. Commun. 4:2054 doi: 10.1038/ncomms3054 (2013). 